\title{
Erratum zu: Theoriebildung in der Kommunikationswissenschaft
}

\section{Eine Bestandsaufnahme zu Bedeutung, Arten und Verfahren der Theorieentwicklung}

\author{
Sebastian Hagen $\cdot$ Felix Frey $\cdot$ Sebastian Koch
}

Online publiziert: 31. Juli 2015

(C) Springer Fachmedien Wiesbaden 2015

\section{Erratum zu: Publizistik (2015) 60:123-146 \\ DOI 10.1007/s11616-015-0229-5}

Durch eine technische Panne ist in der vorigen Ausgabe in der Zusammenfassung des Aufsatzes „Theoriebildung in der Kommunikationswissenschaft“ eine Zeile entfallen. Für diesen Fehler bitten wir die Autoren wie unsere Leser um Entschuldigung. Hier noch einmal der vollständige und korrekte Text der Zusammenfassung:

„Zusammenfassung Methodologisch kommt der Theorieentwicklung eine zentrale Stellung im wissenschaftlichen Erkenntnisprozess zu. Im Gegensatz zu Theorien selbst und der Methodik ihrer Prüfung werden der Prozess und die Verfahren der Theoriebildung in der akademischen Ausbildung und im Fachdiskurs bisher aber kaum thematisiert und reflektiert. Eine solche methodologische Reflexion bietet jedoch große Verbesserungspotentiale für die Praxis der Theorieentwicklung und deren Resultate - die Theorien unseres Fachs.

Dieser Beitrag arbeitet Phasen sowie wichtige Arten und Verfahren des Theoriebildungsprozesses heraus, um im Anschluss inhaltsanalytisch, anhand von jeweils 23 Ausgaben der Fachzeitschriften Publizistik und Medien \& Kommunikationswis-

Die Onlineversion des Originalartikels ist unter doi:10.1007/s11616-015-0229-5 zu finden.

\footnotetext{
S. Hagen, M.A. $(\bowtie)$

Leipzig, Deutschland

E-Mail: seb.hagen@googlemail.com

F. Frey, M.A.

München, Deutschland

S. Koch, M.A.

Leipzig, Deutschland
} 
senschaft aus den Jahren 2003-2012, die Praxis der Theorieentwicklung in unserem Fach zu beleuchten. Dabei wird deutlich, dass der Theorieentwicklung eine große Bedeutung beigemessen wird; allerdings steht die Arbeit mit bereits vorhandenen Theorien im Mittelpunkt.“" 